\title{
ALGORITMO PARA RECONSTRUÇÃO DA IMAGEM DA VAGAROSIDADE A PARTIR DE SINAIS SÍSMICOS CORROMPIDOS COM RUÍDO GROUND ROLL
}

\author{
Cláubio Landney Lima Bandeira \\ Mestrado em Ciência da Computação. Universidade do Estado do Rio Grande do Norte \\ (UERN) Universidade Federal Rural do Semi-Árido (UFERSA). E-mail: \\ claubiobandeira@gmail.com \\ Marcelino Pereira dos Santos Silva \\ Departamento de Informática Universidade do Estado do Rio Grande do Norte (UERN) \\ Mossoró - RN - Brasil. E-mail: marcelinopereira@ uern.br \\ Harold Ivan Angulo Bustos ${ }^{2}$ \\ Departamento de Informática Universidade do Estado do Rio Grande do Norte (UERN) \\ Mossoró - RN - Brasil. E-mail: haroldivan@ hotmail.com
}

\section{RESUMO.}

Inversão sísmica é uma técnica de imageamento sísmico tomográfico para criar um modelo no espaço da velocidade ou da vagarosidade que pode reconstruir corretamente o conjunto de dados sísmicos medido. Isso geralmente é implementado através da minimização de um algoritmo de inversão dos mínimos quadrados. Este algoritmo reconstrói imagens sísmicas com artefatos produzidos pelo ruído impulsivo coerente ground roll contidos no conjunto de dados de entrada sísmicos brutos. Todavia, este ruído compromete a fidelidade dos dados. Apresentamos resultados superiores aplicando filtros visando remover os efeitos causados por este ruído.

PALAVRAS -CHAVE: Inversão sísmica, algoritmo de inversão, ground roll

\begin{abstract}
.
Seismic inversion is a technique of tomographic seismic imaging to create a model in velocity or slowness space that can correctly reconstruct the measured seismic dataset. This is usually implemented by minimizing a least squares inversion algorithm. This algorithm reconstructs seismic images with artifacts yield by impulsive ground roll coherent noise contained in the input raw seismic dataset. However, this noise compromising the reliability of data. We present superior results apllying filters seeking to remove the effects caused by this noise.
\end{abstract}

KEY-WORDS: Sismic inversion, square inversion algorithm, ground roll. 


\section{PARTIR DE SINAIS SÍSMICOS CORROMPIDOS COM RUÍDO GROUND ROLL}

\section{INTRODUÇÃO}

O método sísmico corresponde a uma das ferramentas mais importantes usada na lucrativa indústria de hidrocarboneto (petróleo e gás). Este por sua vez, é utilizado no processo de geração de dados, como por exemplo, os utilizados neste trabalho. Dentre os recursos existentes que objetivam facilitar o estudo deste método estão os softwares de processamento sísmicos que, na sua grande maioria, são proprietários e bastante caros. Porém, visando contornar essa situação, softwares poderosos de distribuição livre e código aberto são desenvolvidos no mundo inteiro. Entre eles está o Madagascar, uma das bibliotecas de processamento de sinais e imagens sísmicas mais importante já desenvolvidas e que, por ser distribuído maneira livre, passou-se a utilizá-lo amplamente em todo o mundo.

Neste artigo, foi utilizado um programa chamado no ambiente Madagascar de cgscan. Esse programa é utilizado para gerar a inversa, ou seja, o campo de vagarosidade sísmica, também conhecida com lentidão. Para isso, ele utiliza o método iterativo das direçõesconjugrado e realiza a inversão após 10 iterações. A vagarosidade corresponde ao inverso da velocidade sísmica e permite a visualização do mapa de velocidades das rochas porosas, o qual constitui um mapa estrutural dos poros no reservatório explorado.

O processo de aquisição de dados sísmicos mapeia tanto as feições de interesse, chamadas de sinais quanto efeitos indesejáveis conhecidos como ruído que compromete o resultado final. Dentre eles está um coerente chamado ruído de rolamento (ground roll), comum em dados sísmicos obtidos em exploração sísmica feita em terra. Recentemente, diversos métodos para eliminá-lo têm sido propostos usando somente filtros (MELO et al., 2009) e outros métodos tais como, Transformada Wavelet (KHENE, 2005). Desta forma, este artigo visa remover este tipo de ruído do espaço da vagarosidade utilizando um conjunto de dados onshore (de terra) do Oriente Médio.

\section{PRINCÍPIOS BÁSICOS ASSOCIADOS À SÍSMICA}

O subsolo é constituído muitas vezes por diferentes tipos de camadas de sedimentos. Essas camadas apresentam propriedades físicas diferentes, dentre elas a elasticidade. Isso faz com que a propagação das ondas também apresente velocidades diferentes, fazendo com que elas, quando encontram meios com essas propriedades, tenham parte de sua energia refletida e outra parte refratada.

Desta forma, surge a prospecção sísmica que baseia-se em medir, em diferentes pontos, o tempo de percurso de ondas elásticas introduzidas artificialmente geradas num ponto próximo à superfície do terreno ou no mar. Estas ondas podem ser geradas por diferentes meios, tais como explosivos, vibradores e canhões de ar comprimido. Devido às descontinuidades elásticas das rochas, partes dessas ondas retornarão a outros pontos da superfície onde serão captadas por sensores (na terra pelos geofones e na água pelos hidrofones). A esta técnica, dar-se o nome de sísmica da reflexão que é o principal método usado na prospecção de hidrocarboneto.

Segundo Robison e Treitel (1980), a exploração de hidrocarbonetos baseada em sísmica 
pode ser dividida em três etapas principais: aquisição, sendo a fase que compreende o levantamento (registro das medidas) dos dados; processamento, visando a produção de imagens da parte interna da terra que sejam mais exatas e aproximadas das camadas geológicas; e interpretação, onde se faz uma análise das imagens obtidas para localizar feições de interesses presentes nelas.

$\mathrm{Na}$ fase de processamento, utiliza-se ferramentas para que se possa gerar modelos de forma confiável e automatizada. Esta é uma das etapas que mais se beneficia com o uso de softwares específicos, estes denominados softwares de processamento sísmico.

\section{SOFTWARE DE PROCESSAMENTO SÍSMICO}

Os softwares de processamento sísmico constituem uma das mais importantes ferramentas utilizadas por empresas petrolíferas. Estes programas geralmente são proprietários, com preços bastantes altos, onde somente empresas que possuam um alto poder aquisitivo podem adquiri-los. Enquanto isso, as instituições de ensino e pesquisa ficam fora desse grupo. Todavia, surgem softwares poderosos de distribuição livre e código aberto e, entre eles, está uma dessas bibliotecas de processamento de sinais e imagens sísmicas: o Madagascar.

O Madagascar é um pacote de programas livre multiplataforma distribuído sobre licença padrão GPL (Licença Pública Geral, do inglês General Public License) de código aberto, para processamento de imagens e dados sísmicos, permitindo trabalhar com dados multidimensionais e experimentos computacionais reprodutíveis. Iniciado em 2003 pelo Dr. Sergey Fomel da BEG (Bureau of Economic Geology) da University of Texas at Austin, somente em 2006 ele foi liberado, contando hoje com mais de 300 programas principais, 3000 testes, documentos reprodutíveis e papers.

Assim como outros pacotes para processamento de imagens e sinais sísmicos, tais como o Seismic Unix (SU) e o Stanford Exploration Project Library (SEPlib), os programas do Madagascar podem ser executados através de linha de comandos. No entanto, elas tornam o trabalho rotineiro e cansativo. Assim, o Madagascar provê uma alternativa para elas, fazendo uso de um construtor denominado SCons (Software Construction), que é um pacote com código-fonte aberto para construção de software, sendo que seus arquivos estão dentro de scripts chamados SConstructs escritos em Python, uma linguagem de programação dinâmica orientada a objeto.

\section{RESULTADOS}

Os resultados deste artigo, foram obtidos a partir um conjunto de dados onshore (de terra) recolhidos em pesquisas de reservatórios petrolíferos no Oriente Médio (GUITTON e SYMESZ, 2003), os quais fazem parte da base de dados do Madagascar. Estes resultados são de grande relevância para a pesquisa em sísmica no Rio Grande do Norte, uma vez que quase todas as pesquisas em sísmica no estado são direcionadas para a sísmica em terra. Neles, são realizados um teste numérico da inversão da transformada dos mínimos quadrados usando os dados onshore e o programa cgscan para processar e encontrar um modelo invertido desses dados. As Figuras 1, 2, 3 e 4 mostram os resultados da inversão após 10 iterações do algoritmo direção-conjugado ponderado (FOMEL, 1996). 
A Figura 1 exibe o conjunto de dados sísmicos de entrada brutos, isto é, sem qualquer processamento realizado sobre eles. Já a Figura 2 mostra a inversão dos mínimos quadrados do conjunto de dados de entrada gerando um modelo de vagarosidade. Nela, podemos identificar alguns artefatos de imagem semelhante faixas retas (no canto superior direito da imagem) e outros artefatos semelhante à curvas. Estes artefatos de imagem estão associados às múltiplas, especificamente à um ruído coerente chamado ruído de rolamento (ground roll) comum em dados sísmicos obtidos em exploração sísmica feita em terra e que tem como características a presença de grandes amplitudes e baixas frequências. $\mathrm{O}$ evento de velocidade principal, associado às ondas sísmicas primárias (ondas de interesse) vindas da subsuperfície até os sensores sísmicos na superfície, está à esquerda desta imagem ao longo de um "rasto" que cruza toda a imagem e é mascarado com faixas horizontais, causada pelas anomalias de amplitudes à curto offset.
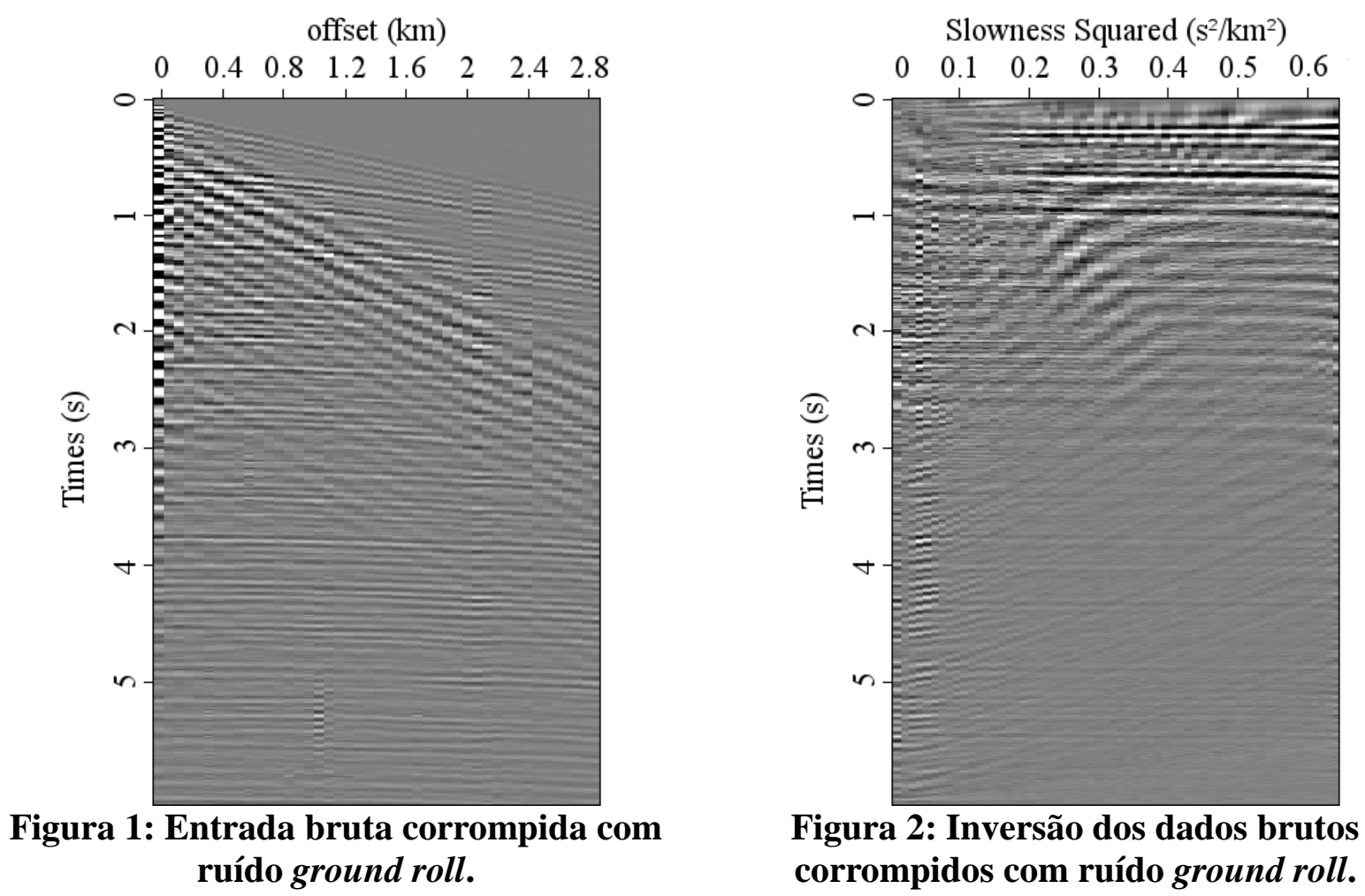

Por fim, a Figura 3 mostra o conjunto de dados modelado a partir da inversão dos mínimos quadrados contaminada com o ruído ground roll. Já a Figura 4 exibe o residual Euclidiano entre os dados modelados (Figura 3) e os dados brutos de entrada (Figura 1). Esta norma rejeita parte do ruído ground roll, porém esse resultado não é o ideal, já que o ruído ground roll interfere na perfeita interpretação dos dados. 


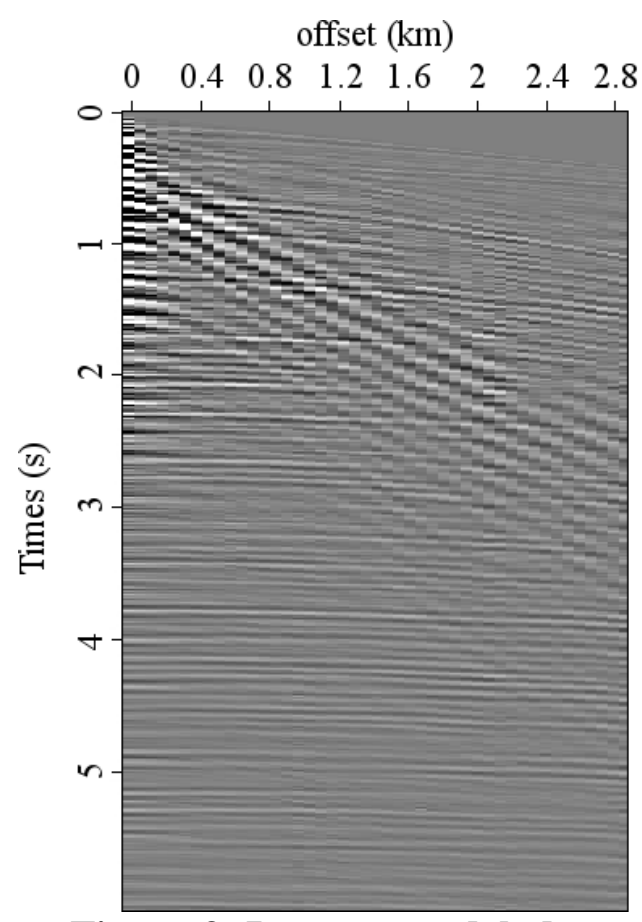

Figura 3: Imagem modelada.

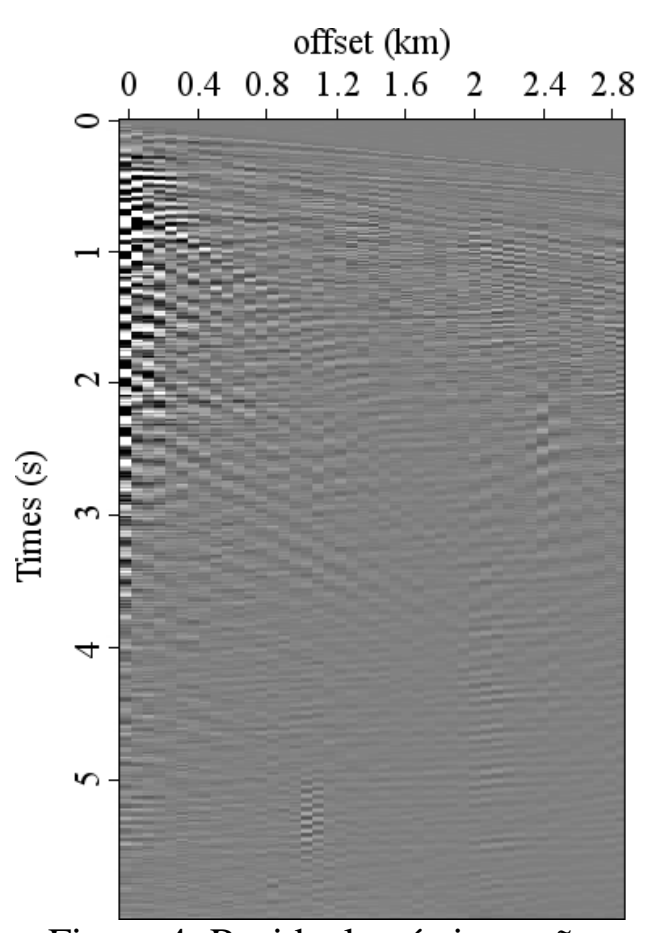

Figura 4: Residual após inversão.

Assim, visando remover esses sinais indesejáveis presentes no espaço da vagarosidade e associados ao ruído ground roll, aplicamos um filtro mediano chamado no Madagascar de despike 2 sobre o conjunto de dados sísmicos de entrada e os filtros bandpass (passa-banda) e imple2, respectivamente, durante a formação da imagem invertida. Este último é responsável por aplicar difusão anisotrópica formulada por Perona e Malik (1990), sendo indicado para arquivos nos quais se quer obter a localização cuidadosa dos dados relevantes da imagem.

Assim, as Figuras 5, 6, 7 e 8 a seguir mostram os resultados da inversão após 10 iterações do algoritmo tendo como entrada, a imagem processada com o filtro mediano (Figura 5). A Figura 6 mostra a inversão dos mínimos quadrados do conjunto de dados de entrada gerando um modelo de vagarosidade a partir da imagem regularizada e com a aplicação dos filtros. Nela é aplicado o filtro passa-banda com uma frequência alta para eliminar adicionais artefatos de imagem que estão na baixa frequência do ruído ground roll (LONDOÑO et al., 2005). Isto regularizou o modelo de vagarosidade que apresenta-se mais limpo, permitindo realçar somente as características associadas com a energia primária das ondas sísmicas. Podemos notar que, a aplicação dos filtros tanto na entrada, quanto no processo de inversão da imagem, eliminou completamente as características associadas ao ruído coerente ground roll do espaço da vagarosidade. 

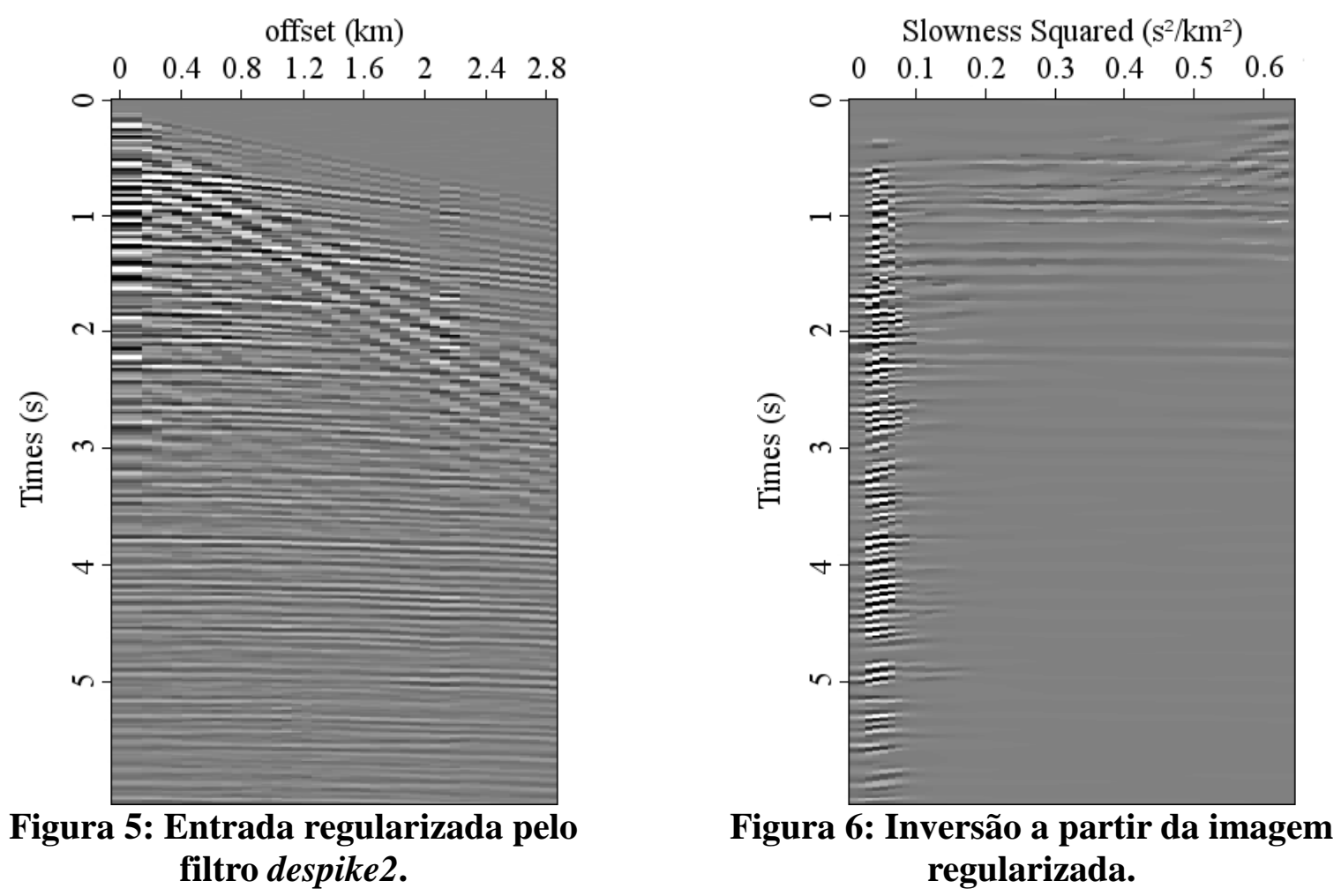

Por fim, a Figura 7 representa a imagem modelada a partir da inversão dos mínimos quadrados usando regularização não-quadrática e aplicando o filtro passa-banda de alta frequência, ambos diretamente no espaço de vagarosidade. Já a Figura 8 exibe o residual Euclidiano entre os dados modelados (Figura 7) e os dados de entrada filtrados pelo filtro mediano (Figura 5). Esta norma exibe características do sinal de entrada associadas ao ruído ground roll indicando que este ruído impulsivo é rejeitado pelo algoritmo desenvolvido neste trabalho.

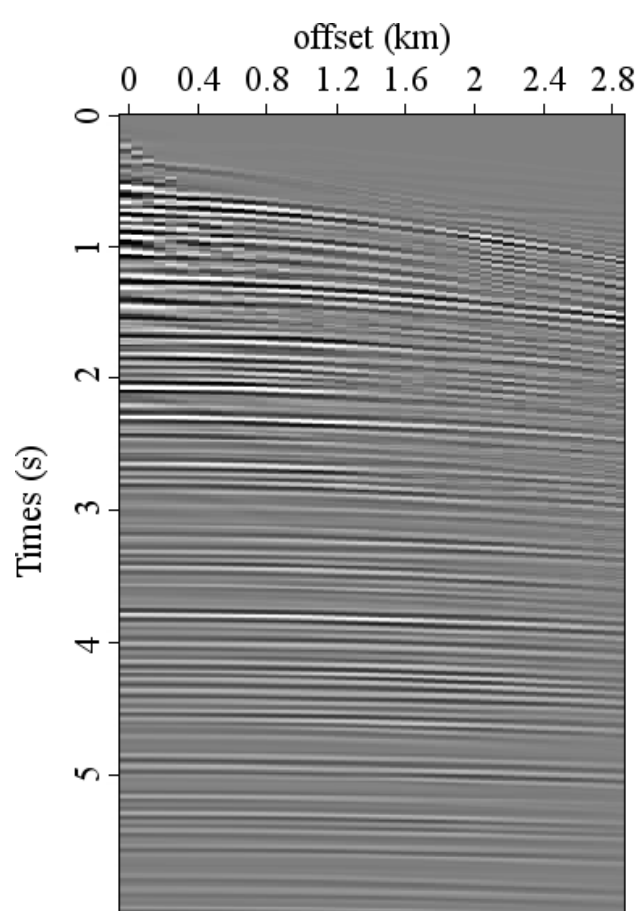

Figura 7: Imagem modelada.

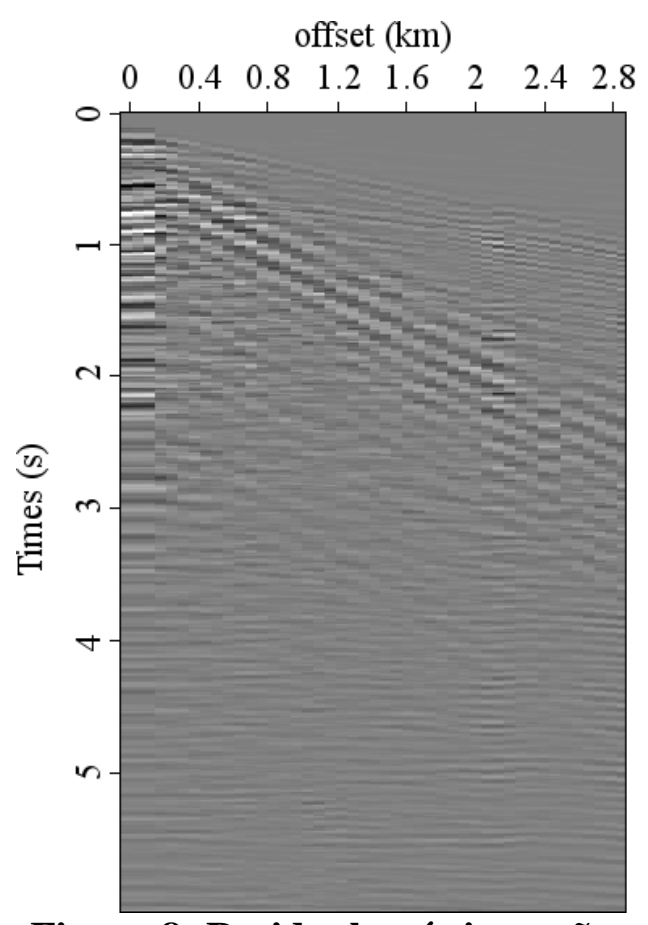

Figura 8: Residual após inversão. 


\section{CONCLUSÃO}

Os dados utilizados neste trabalho refletem uma alta importância para o Rio Grande do Norte, uma vez que este constitui o maior produtor de petróleo em terra do Brasil. Para eles foi proposto um rotina para a remoção dos efeitos indesejáveis causados pelo ruído ground roll. O algoritmo apresentado removeu os artefatos de imagem no modelo de espaço da vagarosidade regularizado associados com ruído coerente ground roll e reforçou feições associadas apenas à energia primária de ondas sísmicas. A aplicação dos filtros utilizados (mediano e passa-banda de alta frequência) foram fundamentais para eliminar os artefatos de imagem no espaço da vagarosidade.

Um outro aspecto relevante deste trabalho, consiste no fato de que desenvolvemos esta aplicação com um pacote de software livre. Desta forma, ele permite que a nossa equipe usem-no em nossas aplicações sem qualquer tipo de restrição, incluindo a possibilidade de transferência de tecnologia para indústrias petrolíferas.

\section{AGRADECIMENTOS}

Os autores agradecem a CAPES e ao CNPq pela concessão das bolsas de pesquisa e pelo apoio financeiro para realização da mesma.

\section{REFERÊNCIAS}

1. FOMEL, S. Least-square inversion with inexact adjoints, method of conjugate direction: a tutorial. Stanford Exploration Project, vol. SEP-92, pp. 253-265, 1996.

2. GUITTON, A., SYMESZ, W. W. Robust inversion of seismic data using the Huber norm. Geophysics, SEG (Society of Exploration Geophysicists), Tulsa, OK, ETATSUNIS (1936) (Revue), vol . 68, n. 4, pp. 1310-1319, July-August, 2003.

3. LONDOÑO, E. G., LOPEZ, L. C., KAZMIERCZAK, T. S. Using the KarhunenLoeve Transform to Suppress Ground Roll in Seismic Data. Earth Sci. Res. J., vol. 9, no. 2, pp. 139-147, December 2005.

4. PERONA, P., MALIK, J. Scale - Space and Edge Detection Using Anisotropic Diffusion. IEEE Transictions on Pattern Analisys and Machine Intelligence, Julho de 1990: 629.

5. ROBINSON, E. a , TREITEL, S. Geophysical Signal Analysis. Prentice-Hall, 1980.

6. MELO, P. E. M., PORSANI, M. J., SILVA, M. G. Ground-roll attenuation using a 2D time-derivative filter. Geophysical Prospecting, 57, 343-353, 2009.

7. KHENE, M. F. Ground-Roll Filtering in the 2-D Wavelet Transform Domain. Land Seismic Forum, SEG 2005, Bahrain 18-21 September 2005. 